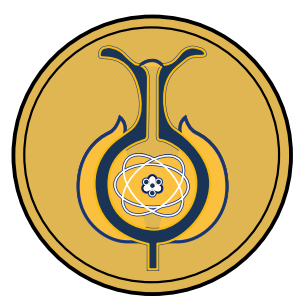

\title{
Estrategia de difusión de la nanotecnología: Enseñanza interdisciplinaria a profesores de educación primaria
}

\author{
Nanotechnology diffusion strategy: interdisciplinary teaching to primary \\ school teachers
}

\section{Estratégia de difusão da nanotecnologia: Ensino interdisciplinar aos professores do ensino fundamental}

\section{Melissa Camacho-Elizondo ${ }^{1}$, Diego Batista-Menezes ${ }^{1}$, Rodrigo Mora-Bolaños ${ }^{1}$, José Roberto Vega-Baudrit ${ }^{1,2}$, Gabriela Montes de Oca-Vásquez}

Received: Feb/5/2021 • Accepted: Jun/8/2021 • Published: Jan/31/2022

\section{Resumen (18)}

El objetivo de la investigación fue socializar los conceptos, aplicaciones y beneficios de la nanotecnología entre personal educador de las diferentes provincias de Costa Rica, para incrementar su aceptación y difusión del tema a los niños y niñas de educación primaria. Se realizó una convocatoria para seleccionar a sujetos educadores de distintas zonas del país. Se impartieron 5 talleres de capacitación en las siguientes localidades: Turrialba, Cahuita, Alajuela, Buenos Aires y Nicoya. En estos se abordaron temas fundamentales de la nanotecnología y sus aplicaciones, así como los aportes que se pueden ofrecer desde el punto de vista educativo. Los talleres se impartieron de manera presencial durante dos días completos, con exposición de charlas, actividades prácticas y discusiones. Además, se preparó material didáctico, como un afiche y un folleto informativo, y dos videos educativos alusivos a la nanotecnología. Se logró capacitar a 98 sujetos educadores, $87 \%$ hombres y $13 \%$ mujeres, de distintas provincias del país. Los resultados de las evaluaciones de los talleres fueron buenos, en términos del conocimiento adquirido con un promedio de calificación de un 57,30\%, comparado con un 5,10\% alcanzado antes de iniciar la capacitación. Este proyecto, contribuyó a fortalecer el conocimiento de los educadores sobre la nanotecnología y sus aplicaciones; además, se dieron a conocer proyectos de investigación nacionales e internacionales relativos a la nanotecnología. Lo anterior permitirá que los educadores sean capaces de transmitir los nuevos conocimientos a la población estudiantil, y se conviertan en multiplicadores del conocimiento.

Palabras clave: Nanotecnología; nanociencia; enseñanza; educación primaria; material didáctico; divulgación científica; conocimiento

\footnotetext{
Melissa Camacho-Elizondo, $\gg$ mcamacho@cenat.ac.cr, (D) https://orcid.org/0000-0003-3553-9041

Diego Batista-Menezes, \dbatista@cenat.ac.cr, (D) https://orcid.org/0000-0002-4993-6171

Rodrigo Mora-Bolaños, \rodrigo.mora@cenat.ac.cr, (D) https://orcid.org/0000-0001-6387-1100

José Roberto Vega-Baudrit, \jvegab@gmail.com, (1D https://orcid.org/0000-0002-2002-1744

Gabriela Montes de Oca-Vásquez, \ gmontesdeoca@cenat.ac.cr, (D) https://orcid.org/0000-0003-2012-816X

1 Laboratorio Nacional de Nanotecnología, Centro Nacional de Alta Tecnología, Costa Rica.

2 Laboratorio de Polímeros, POLIUNA-Escuela de Química Universidad Nacional, Heredia, Costa Rica.
} 


\section{Abstract (1D)}

The objective of the research was to disseminate nanotechnology concepts, applications, and benefits among educators from different Costa Rican provinces to increase their acceptance and dissemination of the subject to elementary students. Teachers from different areas of the country were called to participate. Five training workshops were held in Turrialba, Cahuita, Alajuela, Buenos Aires, and Nicoya covering fundamental nanotechnology concepts and applications, as well as the contributions offered from an educational point of view. The workshops were held in person two full days including talks, practical activities, and discussions. In addition, educational materials were prepared, such as a poster and an information brochure, and two educational videos on nanotechnology. A total of 98 teachers, $87 \%$ men and $13 \%$ women, from different provinces were trained. Workshop evaluations were positive in terms of the knowledge acquired with an average of $57.30 \%$, compared to $5.10 \%$ before starting the training. This project contributed to strengthen teachers' knowledge about nanotechnology and its applications. In addition, national and international nanotechnology research projects were made known. The above will help teachers disseminate new knowledge to the student population, thus becoming knowledge multipliers.

Keywords: nanotechnology; nanoscience; teaching; primary education; teaching material; scientific dissemination; knowledge

\section{Resumo}

Esta pesquisa teve como objetivo socializar os conceitos, aplicações e benefícios da nanotecnologia entre os educadores dos diferentes municípios da Costa Rica, para aumentar a aceitação e difusão do tema nos estudantes do ensino fundamental. Foi realizada uma convocatória para escolher sujeitos educadores de distintas zonas do país. Foram ministradas 5 oficinas de capacitação nas seguintes localidades: Turrialba, Cahuita, Alajuela, Buenos Aires e Nicoya. Foram abordados temas fundamentais da nanotecnologia e suas aplicações, assim como as contribuições que podem ser dadas des de o ponto de vista educativo. Essas oficinas foram ministradas presencialmente durante dois dias completos, com exposição de palestras, atividades práticas e discussões. Além disso, foi preparado material didático, como um cartaz e um folheto informativo, e dois vídeos educativos alusivos à nanotecnologia. Foi possível capacitar 98 sujeitos educadores, $87 \%$ homens e $13 \%$ mulheres, de distintos municípios do país. Os resultados das avaliações das oficinas foram bons, em termos do conhecimento adquirido com uma média de nota de 57,30\%, comparado aos 5,10 $\%$ alcançados antes de iniciar a capacitação. Este projeto contribuiu para a consolidação do conhecimento dos educadores sobre a nanotecnologia e suas aplicações; além disso, divulgaram projetos de pesquisas nacionais e internacionais relativos à nanotecnologia. Isso permitirá que os educadores sejam capazes de transmitir os novos conhecimentos à população estudantil e de se transformar em multiplicadores do conhecimento.

Palavras-chave: Nanotecnologia; nanociência; ensino; ensino fundamental; material didático; divulgação científica; conhecimento

\section{Introducción}

La divulgación de la ciencia y la tecnología representa una forma de comunicación que aglutina diferentes públicos (no especializados y especialistas multidisciplinarios) a temas específicos. Para ello, es cuestión de repasar y saber explicar de forma sencilla lo 
aparentemente complicado (García, 2015). En los últimos años, la nanociencia y la nanotecnología han adquirido mucha relevancia en los ámbitos de la investigación, la divulgación y la educación científica, debido a las numerosos investigaciones y desarrollos que se están produciendo en diferentes campos de importancia social, como su uso para el área de salud mediante el desarrollo de medicamentos de liberación controlada, además de su uso en la ciencia e ingeniería de materiales, y la protección del medio ambiente, entre otros (Meinguer Ledesma, 2019).

La enseñanza de la nanotecnología a nivel educativo es de suma importancia, ya que orienta los avances que genera la ciencia en este campo y fomenta las vocaciones científicas (Castellini et al., 2007). Su enfoque nos permite mostrar la amplitud de aplicaciones científicas y tecnológicas en el área de la nanotecnología en nuestra vida diaria, sin embargo, su enseñanza requiere estrategias que faciliten su difusión, desde la formación de los educadores, el uso de vocabulario de fácil aceptación y la utilización de métodos prácticos, conferencias interdisciplinarias de las diferentes áreas de la nanotecnología, así como sus diversas aplicaciones y posibles conflictos asociados.

Para la década de los años noventa, muchos países comenzaron a desarrollar acciones de divulgación sobre la nanociencia y la nanotecnología, lo cual les permitió contar con estrategias nacionales o regionales; asimismo, algunos países empezaron a incorporar en los programas educativos de ciencias, en los niveles de primaria y secundaria, temas que incluyen conceptos y generalidades de la nanociencia y la nanotecnología (Hsiao-Ping \& Enyi, 2020). Esto, unido a la formación especializada en los niveles de pregrado y posgrado universitario, ha permitido ir creando una cierta cultura en algunos sectores de la población (Tutor et al., 2015). Además, diversas investigaciones se han realizado indicando cómo introducir la nanotecnología a estudiantes en los niveles de primaria y secundaria con buenos resultados en el aprendizaje (HsiaoPing \& Enyi, 2020; Mandrikas et al., 2019; Nandiyanto et al., 2018).

No obstante, en Iberoamérica, en general, no existe una proyección oficial por parte de los Ministerios de Educación locales en cuanto a la inclusión de contenidos de nanotecnología en asignaturas de los ciclos de primaria y secundaria. Otro de los aspectos analizados tiene que ver con los materiales didácticos diseñados para estos niveles educativos. Muchas de las técnicas educativas que se utilizan actualmente dejan de lado la enseñanza de los métodos científicos, sin embargo, con el descubrir y crear, se despiertan, en quienes educan, aptitudes científicas que les permitan desarrollar interés por las ciencias naturales; y de esta forma incentivar entre sus estudiantes la vocación científica (Tutor et al., 2015).

Es un error creer que la ciencia y la tecnología son necesarias únicamente para las ciencias e ingenierías, ya que el mundo avanza en el desarrollo de nuevas tecnologías. Por tanto, es importante transferir conocimientos a miembros de las generaciones futuras, y apoyar el desarrollo de habilidades como: observación, obtención de datos, realización de experimentos sencillos y extracción de conclusiones. Para llevar a cabo esta transferencia de conocimientos es necesario formar a los educadores de forma dinámica, orientándolos en la construcción de conceptos y en el uso de estrategias didácticas prácticas y teóricas, y utilizando analogías comparativas con situaciones cotidianas. Además, es preciso que comprendan las posibilidades asociadas 
a la naturaleza, que puede ser usada como referencia para el desarrollo de proyectos y formación continua (Rodríguez, 2011).

Dada la necesidad e importancia de difundir sobre la nanociencia y la nanotecnología y capacitar a los educadores de I y II ciclo sobre la utilidad e importancia de estas áreas para la sociedad, se creó el proyecto "Nanoprofesor: conceptualización y difusión de la nanotecnología para fomentar la vocación científica en los niños y niñas de educación primaria en Costa Rica" ejecutado por el Laboratorio Nacional de Nanotecnología (LANOTEC) del Centro Nacional de Alta Tecnología (CeNAT), apoyado por el Ministerio de Educación Pública y financiado mediante fondos de la Comisión Costarricense de Cooperación con la UNESCO. El proyecto tuvo como objetivo socializar los conceptos, aplicaciones y beneficios de la nanotecnología entre educadores de las diferentes provincias de Costa Rica para incrementar su aceptación y difusión del tema a los niños y niñas de educación primaria a nivel nacional.

\section{Marco teórico}

\section{La nanotecnología en la educación primaria}

La nanotecnología se refiere a la tecnología que estudia la manipulación y reestructuración de la materia a nivel atómico y molecular, con el objetivo de cambiar sus propiedades y posibilitar aplicaciones innovadoras en diversas áreas. La nanotecnología involucra el diseño, la producción y la aplicación de sistemas físicos, químicos y biológicos en la escala nanométrica, que usa el nanómetro $(\mathrm{nm})$ como unidad de medida $\left(1 \mathrm{~nm}=1 \times 10^{-9} \mathrm{~m}\right)($ ISO/TC 229,2005$)$. Los proyectos de investigación de ciencia y tecnología en nanotecnología prometen avances en áreas tales como materiales y fabricación, medicina y atención médica, energía, tecnología de la información y seguridad nacional (Hsiao-Ping \& Enyi, 2020). La nanociencia y nanotecnología han sido reconocidas como tecnologías emergentes del siglo XXI (Mandrikas et al., 2020).

Es necesario integrar nueva tecnología en la educación o el plan de estudios de I y II ciclo para ayudar al estudiantado a desarrollar sus habilidades y mejorar su curiosidad desde el principio (Xie \& Lee, 2012). Esto puede darle nuevas ideas, y guiarlo a investigar áreas innovadoras, como la nanotecnología. Además, para sostener la investigación y el desarrollo de la nanotecnología en el futuro, muchos países se han apresurado a inyectar el principio de la nanotecnología en la escuela (Hsiao-Ping \& Enyi, 2020; Nandiyanto et al., 2018). Sin embargo, un desafío conceptual importante en el aprendizaje en el área de la nanotecnología y la nanociencia es la capacidad estudiantil para comprender el tamaño real y la escala de los objetos, así como para comprender los principios científicos que subyacen de la nanotecnología y las propiedades únicas del material a nanoescala (Muniz \& Oliver-Hoyo, 2014; Mandrikas et al., 2019). Algunos estudios previos han demostrado que esto podría deberse a la falta de conocimiento por parte del personal docente sobre el tema de nanotecnología, a la falta de recursos y herramientas de aprendizaje y a la carencia de formación profesional relacionada con la enseñanza de la nanotecnología para estudiantes (Hingant \& Albe, 2010). Por lo tanto, se requiere una combinación entre profesorado capacitado y métodos de enseñanza eficaces para encontrar una forma de simplificar las complejidades de la nanotecnología (Nandiyanto et al., 2018). 


\section{Educación primaria en Costa Rica y sus desafíos}

Costa Rica es un país reconocido en América Latina debido a su liderazgo en el tema de educación. Se ha convertido en el primer país de la región en alcanzar una matrícula completa a nivel de primaria; además, el país ha adquirido la responsabilidad de otorgar una educación de calidad en un ambiente positivo para toda la población estudiantil que, poco a poco, se encuentra completando los 9 años de educación básica. También ha asumido la responsabilidad de que sus estudiantes adquieran nuevas habilidades (OCDE, 2017).

Según el Séptimo Informe del Estado de la Educación 2019, presentado por el programa Estado de la Nación, muchos de los centros educativos que imparten enseñanza primaria no brindan el plan de estudios de manera completa, lo cual provoca que en la educación que reciben los niños y las niñas en las aulas exista una gran brecha con las medidas que exige el Ministerio de Educación Pública en las reformas curriculares. La falta de integración de nuevas metodologías de aprendizaje da como resultado que la diferencia entre las prácticas docentes actuales y las metas del país se vuelva significativa. Por otro lado, la mejora en la calidad de la educación que debe realizarse en varias escuelas en zonas rurales del país es motivo de preocupación, debido a los resultados tan bajos que obtienen sus estudiantes.

Cabe mencionar que, a pesar del aumento de la inversión en educación, la brecha de logros entre niñez de países en vías de desarrollo y los de países desarrollados sigue siendo persistentemente alta. Debido a esto, el sector educativo en Costa Rica necesita una reforma más fuerte y más estratégica para abordar estos retos y así asegurarse de que la educación siga siendo un motor para el desarrollo del país (OCDE, 2017).

La ciencia, tecnología, ingeniería y matemáticas (STEM) juegan un papel muy importante en la prosperidad a largo plazo de todas las naciones (Comité Nacional de Ciencias, [NSB, por sus siglas en inglés], 2010). La NSB recomendó que las oportunidades de STEM para todos los estudiantes deberían comenzar desde los grados de primaria e incluir aprendizaje basado en la investigación, colaboración entre compañeros y resolución abierta de problemas del mundo real (Robinson et al., 2014).

Para Costa Rica uno de los grandes desafíos que tiene es invertir en una reforma educativa que logre incentivar las habilidades STEAM de manera temprana en los niños y niñas, con el propósito de lograr mayor inserción en el mercado laboral, ya que en los últimos años el país ha pasado por un gran cambio en su economía, debido a una mayor demanda de personal con alta capacitación. Cabe mencionar que, en Costa Rica, la primera experiencia de trabajo de STEAM, realizada en el 2016, generó puentes de crecimiento para el país, por lo que a partir del 2019 se incluyó la estrategia de educación STEAM en el Plan Nacional de Desarrollo e Inversión Pública (PNDIP) 2019-2022.

\section{Metodología}

La investigación tiene un enfoque mixto cuantitativo y cualitativo (Leavy, 2017). La población de estudio fueron educadores de I y II ciclo pertenecientes a escuelas participantes de la estrategia STEAM (ciencia, tecnología, ingeniería, arte y matemáticas, por sus siglas en inglés) de distintas zonas geográficas de Costa Rica, con una muestra de 98 educadores. La selección de la muestra 
fue de tipo no aleatoria. La selección de los educadores estuvo sujeta a su elección por parte de los asesores regionales de cada zona geográfica donde pertenecía cada escuela participante, para ello, se realizó una coordinación que involucró varios departamentos del Ministerio de Educación Pública, entre ellos, el área de Asesoría Nacional de Ciencias del Departamento de Orientación Educativa y Vocacional; la Dirección de Asuntos Internacionales y Cooperación de I y II ciclo, y el Departamento de Orientación Educativa y Vocacional de la Dirección de Vida Estudiantil. En la Tabla 1, se muestran las escuelas seleccionadas por cantón y provincia donde se impartieron los talleres de capacitación. Dentro de cada escuela sede, asistieron distintos educadores de otras escuelas de la misma zona. Se realizaron en total 5 talleres de capacitación durante el año 2019.

La recolección de datos se realizó mediante listas de asistencia y observación. Los instrumentos aplicados fueron una prueba inicial en el primer día del taller, con el fin de conocer de manera general las bases que tenían los profesores sobre el tema y posteriormente, después de recibir la capacitación se aplicó la misma prueba con el fin de valorar el conocimiento obtenido después de los talleres. Se utilizó el programa estadístico IBM SPSS (versión 23.0; SPSS Inc, Chicago, IL, EEUU) para realizar el análisis de los resultados. Las preguntas de evaluación fueron las siguientes:
1. ¿Qué es la nanotecnología y la nanociencia?

2. Cite y explique algunas aplicaciones de la nanotecnología

3. ¿Conoce usted algún laboratorio de nanotecnología en Costa Rica?

4. Explique alguna investigación en nanotecnología realizada en Costa Rica

Los talleres de capacitación fueron de dos días completos, y durante cada uno de los talleres se desarrollaron 8 charlas divididas en los siguientes temas:

1. Nanotecnología en Costa Rica

2. Introducción a la nanotecnología

3. Nanotecnología y salud

4. Nanotecnología y alimentos

5. Nanotecnología, agricultura y ambiente

6. Nanotecnología y mecatrónica

7. Técnicas de caracterización de los nanomateriales

8. Legislación en nanotecnología

Los temas de las charlas se eligieron con base en los siguientes criterios: conceptos básicos de la nanotecnología y nanociencia, principales áreas de aplicación de la nanotecnología, la experiencia en investigación de los investigadores del Laboratorio Nacional de Nanotecnología (LANOTEC), la interdisciplinariedad y la pertinencia de las charlas de acuerdo con el programa de ciencias de primaria del Ministerio de Educación Pública

Tabla 1. Escuelas por cantón y provincia en donde se realizaron los talleres de capacitación 2019

\begin{tabular}{ll}
\hline \multicolumn{1}{c}{ Escuela } & \multicolumn{1}{c}{ Cantón, Provincia } \\
\hline Escuela Mariano Cortés Cortés & Turrialba, Cartago \\
Escuela Excelencia Cahuita & Cahuita, Limón \\
Dirección Regional de Educación Alajuela & Alajuela, Alajuela \\
Escuela las Lomas & Buenos Aires, Puntarenas \\
Escuela Leonidas Briceño & Nicoya, Guanacaste \\
\hline
\end{tabular}

Nota: Fuente propia de la investigación. 
de Costa Rica. Las charlas se desarrollaron en periodos de 30 a 60 minutos, durante los cuales se mantuvo constante interacción entre los educadores y los expositores, mediante consultas o comentarios de los temas, así como evacuación de dudas que surgieron durante las presentaciones. Las charlas se desarrollaron con base en información obtenida de artículos científicos, libros, y la base de datos de la página web http://statnano.com, para obtener información actual del mercado existente de productos nanotecnológicos a nivel mundial.

Durante las actividades de capacitación, se utilizaron diferentes métodos didácticos para aumentar la tasa de aprendizaje de los participantes, principalmente, porque a través de ellos se dará la difusión de conocimiento hacia los niños y niñas. Los participantes se dividieron en cuatro grupos de trabajo, cada uno guiado por uno de los expositores con el objetivo de que cada grupo realizara una actividad práctica distinta que, posteriormente, fue expuesta a sus compañeros, creando discusiones durante las exposiciones. Por otra parte, se desarrollaron diferentes actividades prácticas, donde los participantes lograron observar experimentos de los temas aprendidos de forma creativa, dinámica y visual. En la Tabla 2 se muestran las prácticas realizadas.

Por otra parte, se realizó un afiche y un folleto informativo, tanto en digital como en físico. Este material incluyó información sobre el proyecto, definiciones sobre la nano escala, la nanotecnología y sus aplicaciones. También, se realizaron dos videos informativos dirigido a niños y niños de I y II ciclo, que refuerzan la temática tratada, los cuales fueron entregados al Ministerio de Educación Pública para su posterior distribución a los diferentes centros educativos.

Tabla 2. Descripción de las actividades prácticas desarrolladas durante los talleres de capacitación 2019

\begin{tabular}{ll}
\hline \multicolumn{1}{c}{ Temas de las actividades prácticas } & \multicolumn{1}{c}{ Descripción } \\
\hline Superficies hidrofóbicas e hidrofílicas & $\begin{array}{l}\text { La práctica se realizó a partir de los conceptos de superficies } \\
\text { hidrofílicas e hidrofóbicas. Cada grupo realizó el mismo } \\
\text { experimento, donde recolectaron materiales del entorno local } \\
\text { (piedras, ramas, hojas, madera) para identificar qué tipo de } \\
\text { propiedades tenían esos materiales (hidrofílicas o hidrofóbi- } \\
\text { cas). Posteriormente y guiados por uno de los expositores del } \\
\text { taller, los grupos recibieron aerosoles que tenían la capacidad } \\
\text { de alterar las superficies de los materiales alterando sus propie- } \\
\text { dades hidrofílicas o hidrofóbicas. }\end{array}$ \\
Noticias nacionales e internacionales sobre & $\begin{array}{l}\text { En grupos se asignaron dos noticias de temas sobre apli- } \\
\text { caciones de la nanotecnología en las áreas de alimentos, } \\
\text { nanotecnología }\end{array}$ \\
& $\begin{array}{l}\text { ambiente, salud y textiles. Cada grupo contó con un espacio } \\
\text { de tiempo para leer las noticias y planear una exposición o dra- } \\
\text { matización sobre el tema asignado. Posteriormente se realizó } \\
\text { retroalimentación por parte de los otros compañeros. } \\
\text { A cada grupo se le asignó una práctica experimental haciendo } \\
\text { uso del Kit comercial "Nano School Box". Se asignaron las } \\
\text { prácticas: superficies conductoras, nanopartículas de ferroflu- } \\
\text { ido magnético, nanopartículas ignífugas y nanomateriales con } \\
\text { memoria de forma. }\end{array}$ \\
\hline Usos del Kit comercial "Nano School Box
\end{tabular}

Nota: Fuente propia de la investigación. 


\section{Análisis y resultados}

Durante la ejecución del proyecto se realizaron 5 talleres de capacitación con la participación de 98 educadores de I y II ciclo de las regiones, Huetar Atlántico (Limón centro), Chorotega (Nicoya), Pacífico central (Puntarenas), Brunca (Buenos Aires) y Central (Alajuela, Cartago). En la Figura 1 se observa el porcentaje de educadores capacitados por provincia y por género. En total se capacitaron un $87 \%$ de mujeres y un $13 \%$ de hombres. Lo anterior confirma lo divulgado por el sexto informe del Estado de la Educación, del Programa Estado de la Nación 2017, donde se indica que, según el Ministerio de Educación Pública, para I y II ciclo en el año 2016 había
47301 funcionarios asignados, de los cuales el $76,8 \%$ corresponde a mujeres.

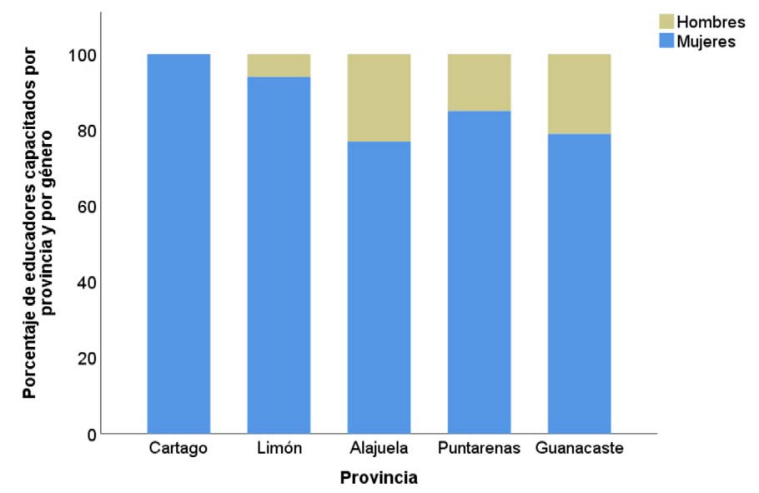

Figura 1. Número total de educadores por género que participaron de los 5 talleres. Nota: Fuente propia de la investigación.

Además, en la Tabla 3 se muestra el número de escuelas por cantón y por

Tabla 3. Número de escuelas por cantón y provincia y número de educadores de primer y segundo ciclo en la Educación General Básica, que recibieron los talleres de capacitación 2019

\begin{tabular}{lcll}
\hline \multicolumn{1}{c}{ Escuela } & Número de educadores & \multicolumn{1}{c}{ Cantón } & Provincia \\
\hline Escuela Mariano Cortés Cortés & 6 & & \\
Escuela Rodolfo Herzog & 5 & Turrialba & Cartago \\
Escuela La Margoth & 5 & & \\
Escuela Jenaro B & 4 & Matina & \\
Escuela Excelencia Estrada & 4 & Limón & Limón \\
Escuela Los Lirios & 4 & Matina & \\
Escuela 28 millas & 5 & Cahuita & \\
Escuela Excelencia Cahuita & 3 & Alajuela & \\
Escuela Juan Rafael Meoño & 4 & Poás & Alajuela \\
Escuela Poasito & 5 & Atenas & \\
Escuela Central de Atenas & 4 & Alajuela & \\
DREA & 4 & Alajuela & \\
Escuela Rincón Chiquito & 4 & & \\
Escuela Concepción & 5 & & \\
Escuela Juan Rafael Mora Porras & 5 & Buenos Aires & Puntarenas \\
Escuela Las Lomas & 4 & & \\
DREGT & 3 & & \\
Escuela Chánguena & 3 & Nicoya & \\
Escuela León Cortés Castro & 4 & Hojancha & Guanacaste \\
Escuela Victoriano Mena & 4 & Nicoya & \\
Escuela Leonidas Briceño & 8 & Nicoya & \\
Escuela El Portal & 4 & & \\
Total & $\mathbf{9 8}$ & &
\end{tabular}

Nota: Fuente propia de la investigación. 
provincia y el número de educadores de primero y segundo ciclo de la Educación General Básica, que recibieron los talleres de capacitación. Es importante mencionar que los educadores que asistieron por zona eran procedentes de diferentes escuelas. Cabe recalcar que Alajuela fue la provincia que presentó más asistencia de educadores de diversas escuelas de la zona, con un $23 \%$ de la cantidad total de los participantes, y fue la Escuela Leónidas Briceño de Nicoya, la que tuvo la mayor cantidad de educadores asistentes, para un total de 8 de los 98 educadores participantes. Por el contrario, Limón fue la provincia en donde se contó con la menor asistencia (18\% del total), a pesar de que la convocatoria se realizó para la misma cantidad de personas.

Asimismo, como parte de las técnicas de aprendizaje, durante los talleres se realizaron demostraciones prácticas sobre diferentes temas de nanotecnología, como prácticas para comprender sobre el efecto loto, superficies conductoras, nanopartículas de ferrofluido magnético, nanopartículas ignífugas y nanomateriales con memoria de forma. Durante esta actividad, los educadores, distribuidos en grupos de trabajo, tuvieron la oportunidad de exponer a todos los participantes una de las prácticas aprendidas, Como resultado se observó que fueron capaces de explicar de una manera muy llamativa y sencilla, las prácticas asignadas a cada grupo, ya que la mayoría de los grupos realizaron dramatizaciones que explicaban alguna aplicación de la nanotecnología. Para las capacitaciones, usamos ideas apropiadas para los educadores de I y II ciclo como "tamaño y escala", "propiedades que dependen del tamaño" y temas más cercanos a la vida cotidiana como el uso de los nanomateriales en la medicina, textiles, medio ambiente, agricultura, construcción, cosméticos, alimentos y electrónica.

Así mismo, se desarrolló un afiche informativo dirigido a niños y niñas de I y II ciclo y un folleto informativo (ver Figura 2) para que los educadores tuvieran información de referencia y apoyo sobre qué es la nanotecnología y sus aplicaciones, los cuales se encuentran disponibles en la página web: http://doi.org/10.5281/zenodo.4768447. Además, se generaron dos videos educativos dirigidos a los niños y niñas de I y II ciclo. El primer video consistió en la definición de qué es la nanoescala o un nanómetro y el segundo en qué es la nanotecnología y sus aplicaciones. Los videos se subieron y están disponibles en la página web del LANOTEC (https://lanotec.cenat.ac.cr).
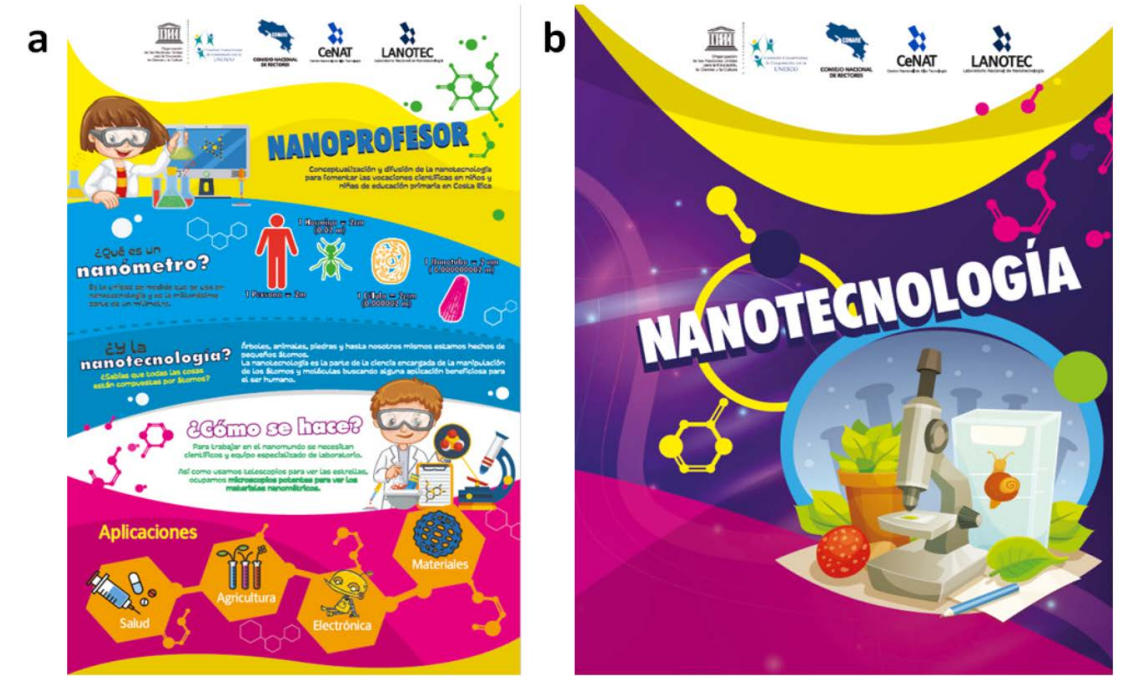

Figura 2. (a) Afiche informativo desarrollado sobre qué es la nanoescala y nanotecnología y (b) folleto informativo desarrollado sobre las aplicaciones de la nanotecnología. 
Los comentarios expresados por algunos de los educadores de secundaria que participaron en los talleres del proyecto "Nanoprofesor" se muestran en la Figura 3. Los educadores mostraron una gran satisfacción por las charlas recibidas.

En términos generales, los resultados de las evaluaciones de los talleres fueron buenos, en términos del conocimiento adquirido durante los dos días de capacitación, con un promedio de calificación de un 57,30 $\%$, comparado con un 5,10\% alcanzado antes de iniciar la capacitación (ver Figura 4), por lo cual llegamos a la conclusión de que los educadores comprendieron conceptos básicos relacionados con la nanotecnología y nanociencia y sus aplicaciones. Estos logros pueden atribuirse al hecho de que los talleres de capacitación incluyeron una amplia gama de actividades como experimentos prácticos, discusiones, lectura de noticias con aplicaciones de la nanotecnología y charlas de capacitación. Este estudio muestra la necesidad de una mayor formación de los educadores en nanociencia y nanotecnología, para aumentar su nivel de conciencia y conocimiento y asegurar su preparación para la enseñanza de este tema, lo cual ha sido reportado de manera similar por otros estudios (Ipek et al., 2020). Es fundamental la formación de los educadores en estos temas de ciencia y tecnología, con el fin de que puedan incentivar a los estudiantes en carreras STEAM, pero para ello poder deben transmitir las materias STEAM como de una manera creativa y emocionante desde su primera introducción, esto para poder lograr una nueva generación de científicos e ingenieros calificados y necesarios para el rápido progreso de la nanotecnología en el mundo (Winkelmann \& Bhushan, 2017). Además, para lograr estos resultados se requiere que los docentes y los investigadores en educación diseñen planes de estudio y programas que demuestren la naturaleza interdisciplinaria de la nanotecnología (Winkelmann \& Bhushan, 2017). Sin embargo, preparar a los docentes para pasar a la enseñanza interdisciplinaria sigue siendo un desafío importante en la actualidad y los profesores necesitan desarrollar tanto habilidades como actitudes hacia la enseñanza interdisciplinaria (Al Salami et al., 2017).

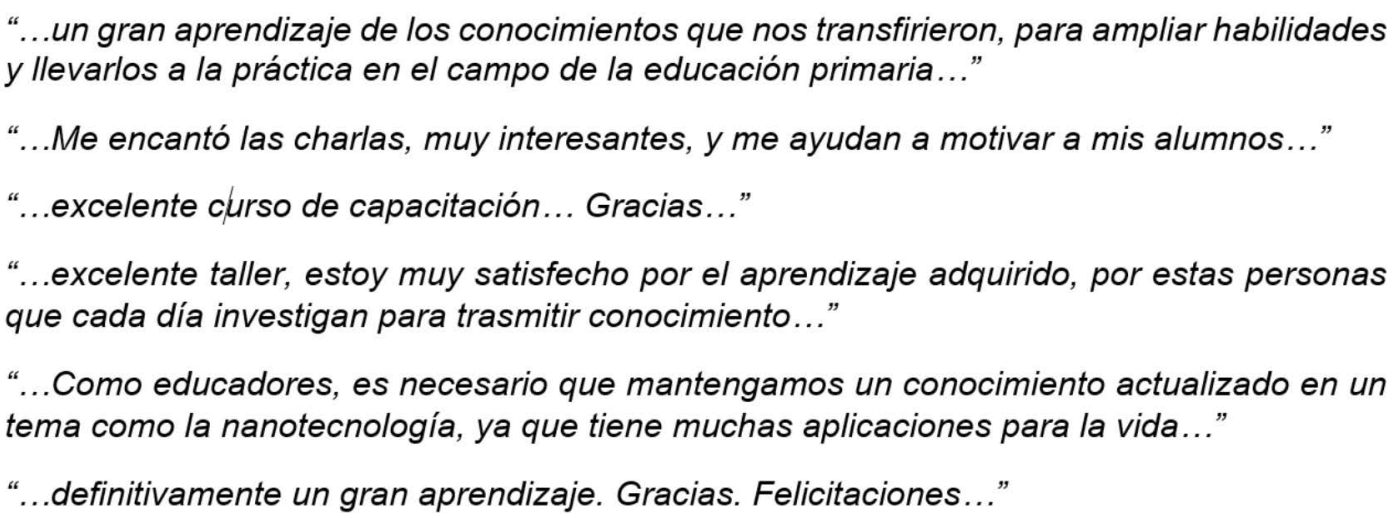

Figura 3. Comentarios expresados por algunos de los participantes en el proyecto "Nanoprofesor: conceptualización y difusión de la Nanotecnología para fomentar la vocación científica en los niños y niñas de educación primaria en Costa Rica”. 


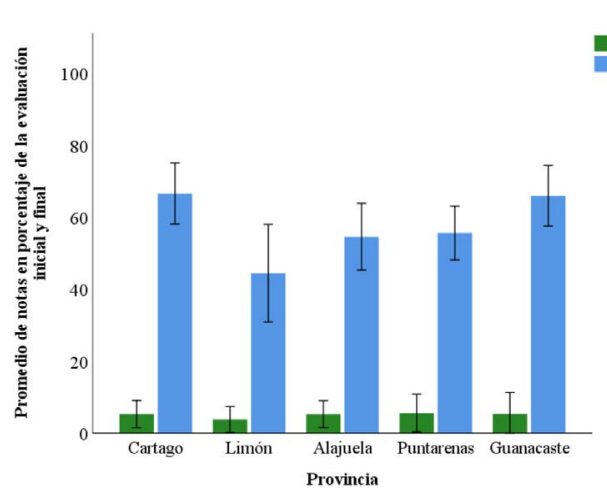

Figura 4. Promedio de notas en porcentaje de la evaluación inicial y final de los educadores participantes por provincia. Los datos se muestran como promedio \pm un error estándar.

Nota: Fuente propia de la investigación.

\section{Conclusiones}

La formación de los educadores es uno de los temas más importantes que se deben abordar para poder tener personal capacitado en áreas de ciencia y tecnología, capaz de transmitir su conocimiento de manera atractiva y de fácil entendimiento a sus estudiantes. En este estudio realizamos talleres de capacitación en diferentes escuelas de las distintas provincias de Costa Rica, utilizando métodos de aprendizaje que incluyeron charlas, prácticas de laboratorio, discusiones grupales y videos. Los temas se abordaron desde una perspectiva interdisciplinaria, con base en el conocimiento y experiencia de profesionales en las áreas de química, biología, biotecnología, ingeniería de materiales, ingeniería química y mecatrónica; lo anterior permitió crear un enfoque holístico para explicar sobre nanociencia y nanotecnología y sus diversas aplicaciones.

El abordaje propuesto para los talleres fue satisfactorio, basado en la opinión de los profesores; se cumplió, así, el objetivo de la divulgación de los conceptos de nanotecnología y sus aplicaciones entre los educadores. Se considera exitosa la mecánica utilizada para las charlas, actividades y material de apoyo, pues, al final de los talleres, los educadores demostraron una mejoría en la definición de nanotecnología, sus aplicaciones y lograron explicar investigaciones realizadas a nivel nacional e internacional.

El aprendizaje por parte de los profesores se ve reflejado también en el alto interés que mostraron en los proyectos presentados relacionados con nanotecnología y sus aplicaciones a nivel nacional e internacional, dados durante las charlas. Esta positiva recepción por parte de los educadores determina su capacidad para convertirse en multiplicadores del conocimiento adquirido durante los talleres.

\section{Financiamiento}

Investigación financiada por la Comisión Costarricense de Cooperación con la UNESCO, proyecto "Nanoprofesor: conceptualización y difusión de la Nanotecnología para fomentar la vocación científica en los niños y niñas de educación primaria en Costa Rica".

\section{Reconocimientos}

Los autores agradecen a la Comisión Costarricense de Cooperación con la UNESCO y al Ministerio de Educación Pública, específicamente a las áreas de Asesoría Nacional de Ciencias del Departamento de Orientación Educativa y Vocacional; la Dirección de Asuntos Internacionales y Cooperación de I y II ciclo, y el Departamento de Orientación Educativa y Vocacional, de la Dirección de Vida Estudiantil. Un especial agradecimiento a las direcciones 
regionales y a los directores de las escuelas participantes por permitir la ejecución de los talleres dentro de las escuelas y dar los respectivos permisos laborales a los educadores, y a Carlos Villalobos, Andrea Rivera, Andrea Chacón y Andrea Araya, del LANOTEC, por sus colaboraciones durante los talleres impartidos.

\section{Conflicto de intereses}

Los autores declaran no tener algún conflicto de interés.

\section{Declaración de la contribución de los autores}

Todos los autores afirmamos que se leyó y aprobó la versión final de este artículo. El porcentaje total de contribución para la conceptualización, preparación y corrección de este artículo fue el siguiente: G.M.V 30\%., M.C.E 30\%., D.B.M 15\%., R.M.B 15\%., J.V.B $10 \%$.

\section{Declaración de disponibilidad de los datos}

Los datos que respaldan los resultados de este estudio serán puestos a disposición por el autor correspondiente [G.M.V], previa solicitud razonable.

\section{Referencias}

Al Salami, M. K., Makela, C. J. \& de Miranda, M. A. (2017). Assessing changes in teachers' attitudes toward interdisciplinary STEM teaching. International Journal of Technology and Design Education, 27(1), 63-88. https://doi. org/10.1007/s10798-015-9341-0

Camacho-Elizondo Melissa, Batista-Menezes Diego, Mora-Bolaños Rodrigo, Vega-Baudrit José Roberto, \& Montes de Oca-Vásquez
Gabriela. (2021, 17 de mayo). Nanoprofessor: Conceptualization and dissemination of the Nanotechnology to promote the scientific vocations in elementary school children in Costa Rica. Zenodo. http://doi.org/10.5281/ zenodo.4768447

Castellini, O., Walejko, G., Holladay, E., Theim, T., Zenner, G. \& Crone, W. (2007). Nanotechnology and the public: Effectively communicating nanoscale science and engineering concepts. Journal of Nanoparticle Research, 9(2), 183-189. https://doi.org/10.1007/ s11051-006-9160-z

Comité Nacional de Ciencias. (2010). Preparing the next generation of STEM innovators: Identifying and developing our nation's human capital (NSB-10-33). Autor.

García, M., \& Foladori, G. (2015). Divulgación de ciencia y tecnología: Los límites del enfoque técnico en las nanotecnologías. Eureka sobre Enseñanza y Divulgación de las Ciencias, 12(3), 508-519. http://dx.doi.org/10.25267/Rev_Eureka_ensen_divulg_cienc.2015.v12.i3.08

Hingant, $\bar{B}$., \& Albe, V. (2010). Nanosciences and Nanotechnologies Learning and Teaching in Secondary Education: A Review of Literature. Studies in Science Education, 46(2), 121-152. https://doi:10.1080/03057267.2010.504543

Hsiao-Ping, Y., \& Enyi, J. (2020). Integrating Nanotechnology in the Science Curriculum for Elementary High-Ability Students in Taiwan: Evidenced-Based Lessons. Roeper Review, 42(1), 38-48. https://doi.org/10.1080/027831 93.2019.1690078

İpek, Z., Atik, A. D., Tan, S. \& Erkoç, F. (2020). Awareness, exposure, and knowledge levels of science teachers about nanoscience and nanotechnology. Issues in Educational Research, 30(1), 134-155.

Leavy, P. (2017). Research design: Quantitative, qualitative, mixed methods, arts-based, and community-based participatory research approaches. Nueva York: Guilford Publications.

Mandrikas, A., Michailidi, E. \& Stavrou, D. (2020). Teaching nanotechnology in primary education. Research in Science \& Technological Education, 38(4), 377-395. https://doi.org/10 $.1080 / 02635143.2019 .1631783$

Meinguer Ledesma, J. (2019). La comunicación de la nanotecnología del carbono a través del análisis crítico de textos informales en la educación química 
preuniversitaria. Mundo nano. Revista interdisciplinaria en nanociencias y nanotecnología, 12(22), 1-33. https://doi.org/10.22201/ ceiich.24485691e.2019.22.61953

Muniz, M. N. \& Oliver-Hoyo, M. T. (2014). On the use of analogy to connect core physical and chemical concepts to those at the nanoscale. Chemistry Education Research and Practice, 15(4), 807-823. https://doi.org/10.1039/ $\mathrm{C} 4 \mathrm{RP} 00097 \mathrm{H}$

Nandiyanto, A. B. D., Asyahidda, F. N., Danuwijaya, A. A., Abdullah, A. G., Amelia, N. I.A., Hudha, M. N. \& Aziz, M. (2018). Teaching "nanotechnology" for elementary students with deaf and hard of hearing. Journal of Engineering Science and Technology, 13(5), 1352-1363.

OCDE, (2017). Informe. Análisis de la OCDE acerca de las políticas nacionales para educación: $\mathrm{La}$ educación en Costa Rica.

Programa Estado de la Nación. (2017). Informe Estado de la Educación ( $6^{\text {ta }}$ ed.) Autor.
Robinson, A., Dailey, D., Hughes, G. \& Cotabish, A. (2014). The effects of a science-focused STEM intervention on gifted elementary students' science knowledge and skills. Journal of Advanced Academics, 25(3), 189-213. https://doi:10.1177/1932202X14533799

Rodriguez, D., \& Ávila, A. (2011). Experiencias en micro y nano escalas para niños y jóvenes. Mundo Nano, 4(2), 121-128. https://doi. org/10.22201/ceiich.24485691e.2011.2.45080

Tutor, J. \& Takeuchi, N. (2015). ¿Por qué es necesaria la divulgación y la formación en nanotecnología?. MOMENTO-revista de fisica, 51, 4558. https://doi.org/10.15446/mo.n51.56204

Winkelmann K. \& Bhushan B. (2017). Global Perspectives of Nanotechnology Education. In Bhushan B. (Eds), Springer Handbook of Nanotechnology. Springer Handbooks. https:// doi.org/10.1007/978-3-662-54357-3_47

Xie, C., \& Lee, H. S. (2012). A visual approach to nanotechnology education. International Journal of Engineering Education, 28(5), 1006-1018.

\section{(c) $(1)(9)$}

Estrategia de difusión de la nanotecnología: Enseñanza interdisciplinaria a profesores de educación primaria (Melissa Camacho-Elizondo $•$ Diego Batista-Menezes $\bullet$ Rodrigo Mora-Bolaños1 • José Roberto Vega-Baudrit1 • Gabriela Montes de Oca-Vásquez) Uniciencia is protected by Attribution-NonCommercial-NoDerivs 3.0 Unported (CC BY-NC-ND 3.0) 